\title{
Post traumatic septic arthritis of the elbow and osteomyelitis of the distal radius in a 12-year-old child
}

\author{
Jamie Hind*, Neil Ashwood, Islam Mubark and Babis Karagkevrekis \\ University Hospital of Derby and Burton (Queens Hospital), Belvedere Road, Burton on Trent, DE13 0RB, United Kingdom
}

\begin{abstract}
Septic arthritis and Osteomyelitis are important conditions that must be excluded in a child presenting with musculoskeletal pain. A delay in diagnosing such conditions may result in significant morbidity and mortality. This is a rare case of a patient that developed ipsilateral concomitant osteomyelitis and septic arthritis of the same limb in a child following a fall.
\end{abstract}

\section{Background}

This case report highlights the importance of diagnosing and managing musculoskeletal pain in children. The majority of musculoskeletal pain in children is soft tissue or bony injuries following a fall, however, inflammatory conditions such as osteomyelitis and septic arthritis must be ruled out. The importance of an accurate diagnosis and prompt management cannot be stressed enough for patient with septic arthritis and osteomyelitis, as a delay in treatment may result in significant morbidity and mortality. We report a rare case where a child developed septic arthritis and osteomyelitis in the same limb following a simple mechanical fall. From this case we can learn the importance of follow up for children with pain of unknown cause and highlight the importance of the of diagnosing and managing septic arthritis and osteomyelitis and the challenges that may arise.

\section{Case report}

A 12-year-old boy presented to the Emergency Department (ED) complaining of pain in the right elbow three days following a mechanical fall on the beach. Apart from the right elbow, there were no other injuries to note. On examination the patient was Apyrexial. There was local tenderness to the right elbow but it was not red or hot to touch. Neurovascular status was normal. A plain radiograph was performed of the right elbow which was normal. The child was discharged home with a backslab cast and a fracture clinic follow up appointment. Five days later, the child attended the fracture clinic appointment, with increasing pain in the elbow, feeling nauseous and generally unwell. On examination, the right elbow was red and associated with a reduced range of movement. There was also swelling of the right forearm and reduced range of movement of the right wrsit. The child was apyrexial and his observations were normal. Blood tests and X-rays of the right elbow and forearm were requested. The C-Reactive Protein (CRP) and White Blood Cell Count (WBC) were within normal limits. The X-ray showed anterior fat pad sign of the elbow (Figure 1). The child was admitted with diagnosis of compartment syndrome and was booked on the emergency list for surgical exploration of right forearm. Later that day, the patient was reviewed in the paediatric ward, with findings of raised local temperature, increased swelling and inability to move fingers. The CRP level was now elevated to 331 and the WBC was
6.9. Intravenous antibiotics were commenced and urgent MRI and ultrasound scans were requested. The next day the patient complained of increased pain when moving arm and temperature spiked at 38.3C. The MRI scan of the right elbow suggested the likelihood of septic arthritis of the elbow and osteomyelitis of the distal radius (Figure 2). Oral fucidic acid was added to the IV flucloxacillin and benzylpenicillin. The following day patient was taken to the operating theatre for a washout of the right elbow and an open decompression and washout of right distal radius. The elbow fluid aspirate consisted of cloudy fluid and pus which grew staphylococcus aureus. The bone edge from the distal radius was sent along for further analysis which later returned confirming osteomyelitis. Post-operatively the patient continued to have temperature spikes and raised inflammatory marker despite the surgery and antibiotics so the patient was then taken to theatre for a second washout of the right elbow and right wrist. Following the second procedure the child started to show signs of improvement. The distal radius bone fragment analysis confirmed osteomyelitis. Repeat MRI scan after surgical washout showed marked synovitis in the elbow but no focal infection. The patient was discharged ten days after the second washout no local or systemic signs of infection. The patient was followed up at one week and one month. At the one month follow up appointment, the patient had return of full range of movement and normal inflammatory markers.

\section{Discussion}

Joint pain is a common presenting complaint in children. The three main groups of differential diagnoses for joint pain include: trauma, malignancy [1] and arthritis either reactive, inflammatory or septic $[2,3]$. The presence of minor trauma does not exclude other causes. This case report highlights the challenges that may arise when faced with a child presenting with joint pain and the importance of follow

${ }^{\star}$ Correspondence to: Jamie Hind, University Hospital of Derby and Burton (Queens Hospital), Belvedere Road, Burton on Trent, DE13 0RB, United Kingdom, E-mail: jamie.hind@nhs.net

Received: August 04, 2020; Accepted: August 11, 2020; Published: August 14 2020 


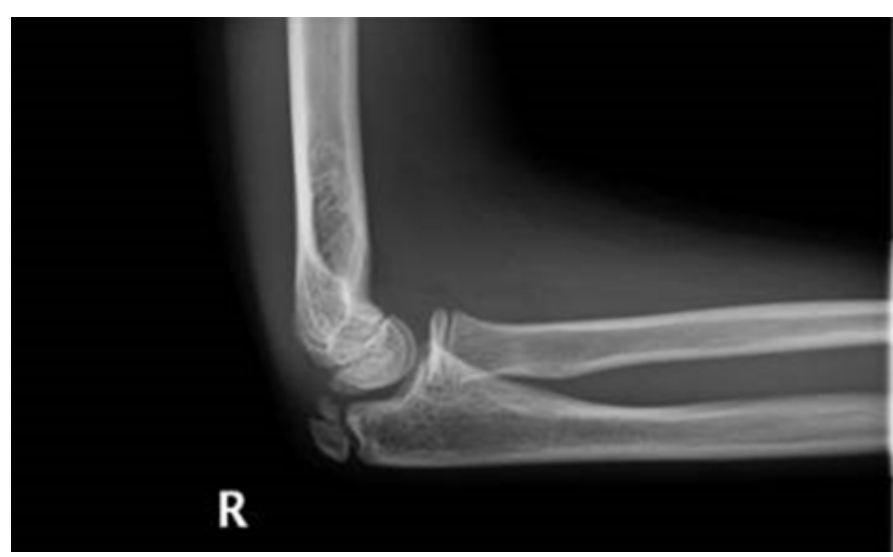

Figure 1. Lateral Xray of the right elbow demonstrating mild anterior fat pad sign
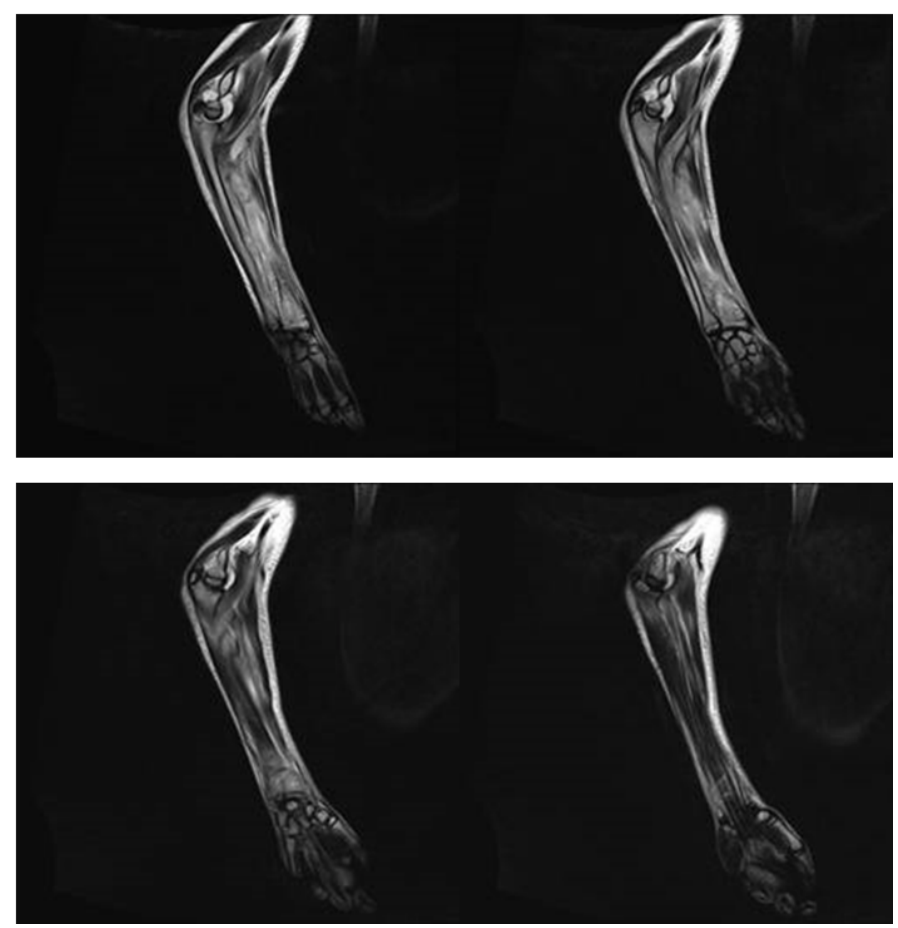

Figure 2. MRI of the right elbow and forearm with evidence of osteomyelitis of the distal radius associated with septic arthritis

up when the cause cannot be identified on admission. In this case, and others alike, the history of trauma may be a distraction, expecting the cause to be the result of bony or soft tissue injury. With the significant morbidity associated with both osteomyelitis and septic arthritis, these conditions must not be missed [4]. Osteomyelitis and septic arthritis must be excluded in any child presenting with musculoskeletal pain and/or systemic symptoms because delayed or inadequate treatment may cause irreversible joint destruction [3-5]. Septic arthritis should always be suspected if a child presents with temperature and acutely hot and swollen joint [6]. The diagnosis is based on findings similar to the Kocher's criteria [7] for hip septic arthritis which include history of fever, refusal to bear weight (pseudoparalysis [6]), ESR $>40 \mathrm{~mm} / \mathrm{hr}$, WBC $>12,000 / \mathrm{uL}$. C-Reactive Protein was added to the criteria at a later time [8]. The history of minor trauma does not exclude the possibility of infection, as in our case, and careful follow up is recommended in all children presenting with joint pain [9]. The imaging studies that can be used for the diagnosis of septic arthritis and osteomyelitis are plain radiographs, bone scans, ultrasound scans and magnetic resonance imaging (MRI) scans. Of all, MRI scan is currently the imaging modality of choice, especially for osteomyelitis [10]. The main MRI findings in septic arthritis are synovial enhancement (98\%), perisynovial oedema (84\%), joint effusion (70\%), fluid outpouching (53\%), fluid enhancement (30\%), and synovial thickening (22\%). Associated osteomyelitis is often diffuse and appears as abnormalities in T1 signal sequences $[11,12]$. Utilizing advanced imaging (CT scan, bone scan, and/ or MRI studies) in patients with septic arthritis may shorten hospital stays, decrease the number of operative procedures required, and possibly limit infectionrelated sequelae by identifying concurrent infections earlier [4]. The most common causative organism is Staphylococcus aureus [13] followed by MRSA and Streptococcus pneumoniae [14]. Hip joint is the one which is usually affected. Knee joint follows in hierarchy, whereas elbow joint is more rarely involved [15]. Early hospital admission, intravenous antibiotic therapy and possibly surgical intervention have been proven to be affected as treatment. Intravenous antibiotics should be administered for a minimum of for 2-4 days followed by 20 days of oral therapy for osteomyelitis or 10 days for septic arthritis $[3,10]$. To the best of our knowledge this is the first delayed onset elbow septic arthritis and radial osteomyelitis that has been reported, with eight days from initial symptoms to laboratory and radiological findings which led to the final diagnosis.

\section{References}

1. Trapani S, Grisolia F, Simonini G, Calabri GB, Falcini F (2000) Incidence of occult cancer in children presenting with musculoskeletal symptoms: a 10 -year survey in a pediatric rheumatology unit. Semin Arthritis Rheum 29: 348-359.

2. Tallen G, Bielack S, Henze G, Horneff G, Korinthenberg R, et al. (2014) Musculoskeleta Pain: A New Algorithm for Differential Diagnosis of a Cardinal Symptom in Pediatrics. Klinische Padiatrie 226: 86-98.

3. Dodwell ER (2013) Osteomyelitis and septic arthritis in children: current concepts Curr Opin Pediatr 25: 58-63.

4. Tanwar YS, Jaiswal A, Singh S, Arya RK, Lal H (2014) Acute pediatric septic arthritis: a systematic review of literature and current controversies. Pol Orthop Traumatol 79: 23-29.

5. De Boeck H (2005) Osteomyelitis and septic arthritis in children. Acta orthopaedica Belgica 71: 505-515.

6. Coakley G, Mathews C, Field M, Jones A, Kingsley G, et al. (2006) BSR \& BHPR, BOA, RCGP and BSAC guidelines for management of the hot swollen joint in adults. Rheumatology 45: 1039-1041.

7. Kocher MS, Zurakowski D, Kasser JR (1999) Differentiating between septic arthritis and transient synovitis of the hip in children: an evidence-based clinical prediction algorithm. J Bone Joint Surg Am 81: 1662-1670.

8. Caird MS, Flynn JM, Leung YL, Millman JE, D'Italia JG, et al. (2006) Factors distinguishing septic arthritis from transient synovitis of the hip in children. A prospective study. J Bone Joint Surg Am 88: 1251-1257.

9. Tina T (2007) Osteomyelitis and septic arthritis. In: Ronald M. Perkin DAN, James D. Swift, editor. Pediatric Hospital Medicine: Textbook of Inpatient Management. Philadelphia: Lippincott Williams \& Wilkins. pp: 471.

10. Kaplan SL (2009) Challenges in the evaluation and management of bone and joint infections and the role of new antibiotics for gram positive infections. Adv Exp Med Biol 634: 111-120.

11. Karchevsky M, Schweitzer ME, Morrison WB, Parellada JA (2004) MRI findings of septic arthritis and associated osteomyelitis in adults. AJR Am J Roentgenol 182: 119-122.

12. Averill LW, Hernandez A, Gonzalez L, Peña AH, Jaramillo D (2009) Diagnosis of osteomyelitis in children: utility of fat-suppressed contrast-enhanced MRI. AJR Am J Roentgenol 192: 1232-1238.

13. Paakkonen M, Peltola H (2013) Bone and joint infections. Pediatr Clin North Am 60 425-436.

14. Young TP, Maas L, Thorp AW, Brown L (2011) Etiology of septic arthritis in children an update for the new millennium. Am J Emerg Med 29: 899-902.

15. Bowakim J, Marti R, Curto A (2010) Elbow septic arthritis in children: clinica presentation and management. J Pediatr Orthop Part B 19: 281-284.

Copyright: (C2020 Hind J. This is an open-access article distributed under the terms of the Creative Commons Attribution License, which permits unrestricted use, distribution, and reproduction in any medium, provided the original author and source are credited. 